\title{
GENERALIZED RIESZ PROJECTIONS AND TOEPLITZ OPERATORS
}

\author{
TAKAHIKO NAKAZI AND TAKANORI YAMAMOTO
}

\begin{abstract}
Let $1<p<\infty$. In this paper, for a measurable function $v$ and a weight function $w$, the generalized Riesz projection $P^{v}$ is defined by $P^{v} f=v P\left(v^{-1} f\right),\left(f \in L^{p}(w)\right)$. If $P_{0}$ is the self-adjoint projection from $L^{2}(w)$ onto $H^{2}(w)$, then $P_{0}=P^{\alpha}$ for some outer function $\alpha$ satisfying $w=|\alpha|^{-2}$. In this paper, $P^{v}$ on $L^{p}(w)$ is studied. As an application, the invertibility criterion for the generalized Toeplitz operator $T_{\phi}^{v}$ and the generalized singular integral operator $\phi P^{v}+Q^{v}, Q^{v}=I-P^{v}$ are investigated using the weighted norm inequality. The operator norm inequality for the generalized Hankel operator $H_{\phi}^{v}$ is also presented.
\end{abstract}

Mathematics subject classification (2000): 42B30, 47B35.

Key words and phrases: weighted norm inequality, weighted Hardy space, Toeplitz operator, Muckenhoupt condition $\left(A_{p}\right)$, Riesz projection.

\section{REFERENCES}

[1] A. BötTCheR AND B. Silbermann, Analysis of Toeplitz Operators (Springer, Berlin, 1990).

[2] R. COIFMAn AND R. ROCHBERG, Projections in weighted spaces, skew projections and inversions of Toeplitz operators, Integral Equations and Operator Theory 5 (1982), 145-159.

[3] F. FoRELLI, The Marcel Riesz theorem on conjugate functions, Trans. Amer. Math. Soc. 106 (1963), 369-390.

[4] J. GaRnetT, Bounded Analytic Functions. (Academic Press, New York, 1981).

[5] I. GOHBERG AND N. KRUPNIK, One-Dimensional Linear Singular Integral Equations. Vols. I,II, (Birkhäuser, Basel, 1992).

[6] B. HollenBeCK AND I.E. Verbitsky, Best constants for the Riesz projection, J. Funct. Anal. 175 (2000), 370-392.

[7] T. NAKAZI, Commutator of two projections in prediction theory, Bull. Austral. Math. Soc. 34 (1986), 65-71.

[8] T. NAKAZI, Kernels of Toeplitz operators, J. Math. Soc. Japan 38 (1986), 607-616.

[9] T. NAKAZI, Toeplitz operators and weighted norm inequalities, Acta Sci. Math. (Szeged) 58 (1993), 443-452.

[10] T. NAKAZI AND T. YAMAMOTO, Norms of some singular integral operators and their inverse operators, J. Operator Theory 40 (1998), 185-207.

[11] N.K. NIKOLSKI, Treatise on the Shift Operator. (Springer, Berlin, 1986).

[12] N.K. NiKOLSKI, Operators, Functions, and Systems. Vols. I,II, (Amer. Math. Soc., 2002).

[13] R. RochBerG, Toeplitz operators on weighted $H^{p}$ spaces, Indiana Univ. Math. J. 26 (1977), 291-298. 